\title{
Depression and Health-Related Quality of Life Among Young Adult Breast Cancer Patients: The Mediating Role of Reproductive Concerns
}

\author{
Ana Bártolo, MSc, ${ }^{1}$ Isabel M. Santos, $\mathrm{PhD},{ }^{2}$ Elisabete Valério, $\mathrm{MSc},{ }^{3}$ and Sara Monteiro, $\mathrm{PhD}^{1}$
}

Biological motherhood plays an important role in the lives of many young women facing breast cancer and threats to reproduction may be disruptive. In this study, we explored the indirect effects of the importance of parenthood and childlessness on depression and health-related quality of life (HRQoL) among cancer patients 18-40 years of age $(n=104)$ through reported reproductive concerns. These specific concerns fully mediated the relationship between the importance of parenthood in women's lives and HRQoL. Greater importance of parenthood was directly associated with higher depression symptoms. Interventions should address the reproductive needs and concerns of patients to improve their HRQoL.

Keywords: parenthood, women, childless, distress, oncology, younger

\section{Introduction}

$\mathbf{C}$ URRENT ESTIMATES INDICATE that breast cancer in young adult women (20-39 years) has incidence rates in European countries ranging from 12.6/100.000 to 41.0/ 100.000, for Moldova and Italy, respectively. ${ }^{1}$ This malignancy is known to influence the reproductive ability through cytotoxic effects of chemotherapy and radiotherapy treatments. Most chemotherapeutic regimes used in breast cancer treatments can induce the damage of germinal tissue in the gonads and lead to premature ovarian failure. ${ }^{2}$ The aging of the reproductive system is another factor affecting the ovarian reserve of those who are nearer to the latter end of young adulthood, when anticancer treatments are completed (e.g., prolonged adjuvant hormonal therapy ${ }^{3}$ ). Therefore, the desire for biological motherhood and potential lack of choice in family building projects may represent additional challenges in the course of the disease.

Specific guidelines have been developed to improve fertility counseling, but some women still report limited conversations, which only focused on warnings not to become pregnant during chemotherapy, ${ }^{4}$ and report a lack of information regarding the early menopause and infertility risk and options to assess and preserve their fertility. ${ }^{5}$ Previous research has identified professional and organizational barriers to discussion of oncofertility care with cancer patients. Most professionals point to lack of time, awareness, and knowledge as barriers to dealing with fertility issues and report disagreement between departments on who is responsible for discussing this topic. ${ }^{6}$ Nevertheless, some patients have not yet completed their families at the time of diagnosis and desire to have children after treatment. ${ }^{7}$

An unfulfilled desire for parenthood seemed to be associated with impaired mental health. ${ }^{8}$ Distress caused by an interrupted desire for childbearing can persist even 5-10 years post-treatment. ${ }^{9}$ Moreover, the wish for children in the future has been identified as a significant positive predictor of the reproductive concerns among women with breast cancer. ${ }^{9,10}$ Menstrual changes, potential treatment-related fertility loss, and fertility options have been consistently indicated as major concerns among younger women. ${ }^{11,12}$ However, other issues related to their own health after a potential pregnancy and the genetic risk to their offspring also worry cancer patients. ${ }^{13}$ These unique concerns reflect a serious problem, since they have been shown to increase the risk of depression ${ }^{14,15}$ and a poorer QoL. ${ }^{16}$

Our aim was to provide a better understanding of the mechanisms involved in the maladjustment of young adult women faced with a reproduction-threatening disease. In general, although the desire for children and childlessness are a burden and a worrying factor, it is unclear how much the meaning of motherhood influences psychosocial outcomes among cancer patients. Thus, we explored the association between the representation of the importance of parenthood,

\footnotetext{
${ }^{1}$ Center for Health Technology and Services Research (CINTESIS), Department of Education and Psychology, University of Aveiro, Aveiro, Portugal.

${ }^{2}$ William James Center for Research, Department of Education and Psychology, University of Aveiro, Aveiro, Portugal.

${ }^{3}$ Breast Clinic, Instituto Português de Oncologia Francisco Gentil, Porto, Portugal.
} 
fertility, and parenthood concerns and depression, and healthrelated quality of life (HRQoL). Furthermore, based on the emergent literature, we hypothesized that the relation between these variables is likely to be complex, that is, greater importance of parenthood in women's lives may be associated with higher depression symptoms and lower HRQoL through reproductive concerns.

\section{Materials and Methods}

\section{Participants and procedure}

This cross-sectional study included breast cancer patients who were recruited from a hospital in the North of Portugal. Women 18-40 years of age, diagnosed over the age of 18 with early stage breast cancer $(\leq$ IIIA) who received chemotherapy were eligible to participate in the study. The researcher invited 170 patients to participate on the day of the medical appointment. Of these, 104 were included in this analysis. We excluded patients for the following reasons: having undergone hysterectomy, prophylactic oophorectomy or tubal ligation procedures, or being currently pregnant. The protocol for this research was approval by the Hospital Ethics Committee and participants gave their consent before enrolment. Sociodemographic and clinical information was assessed by standard questions.

\section{Measures}

Reproductive concerns. Fertility and parenthood concerns were assessed with the 18-item Reproductive Concerns After Cancer Scale (RCACS). ${ }^{17}$ The psychometrically validated Portuguese version of the scale presents five dimensions: fertility potential, children's health risk and future life, partner disclosure, barriers to getting pregnant/having children, and acceptance of the fertility status. ${ }^{18}$ For each item, participants are asked to rate how much they agree/disagree with each statement on a 5-point Likert scale. The global index ranges from 18 to 90 with higher scores indicating higher levels of reproductive concerns. In this study, the Cronbach's alpha coefficient was 0.836 .

Importance of parenthood. Representations of the importance of parenthood were measured using two subscales of the Portuguese version of the Fertility Problem Inventory. Based on the approach proposed by Moura-Ramos et al., ${ }^{19}$ the importance of parenthood global index was obtained from the need for parenthood and rejection of a childfree lifestyle subscales, involving questions related to perceiving parenthood as a main goal in life and the negative view of life without a child. Participants respond using a 6-point Likert scale from "Strongly disagree" to "Strongly agree." A higher score represents greater importance of parenthood in women's lives. The alpha coefficient was good in this sample $(\alpha=0.859)$.

Depression. Depression symptoms were assessed through the depression subscale of the 14-item Hospital Anxiety and Depression Scale (HADS). ${ }^{20}$ This subscale is composed of seven items and participants are invited to respond using a 4-point Likert scale. The total score ranges from 0 to 21 , with higher scores indicating a higher level of depressive symptoms. A Cronbach's alpha of 0.847 was obtained in the present sample.

Health-related quality of life. The European Organization for Research and Treatment of Cancer Quality-of-Life Questionnaire Core-30 (EORTC QLQ-C30) ${ }^{21}$ is a thirty-item questionnaire used to measure HRQoL, including five functional scales, a global health status/QoL scale, threesymptom scales, and single-item measures. In this study, only the functional scales assessing physical, role, emotional, cognitive, and social functioning were used. Items are rated on a 4-point Likert scale ranging from "not at all" to "very much." The scores range from 0 to 100 , with higher scores indicating better functioning. Cronbach's alpha coefficients were acceptable (ranging from 0.605 to 0.920 for cognitive and social functioning, respectively).

\section{Statistical analysis}

Data analysis was performed with IBM SPSS software, version 24 and IBM Amos software, version 24. Pearson's correlations were used to evaluate the bivariate relations between importance of parenthood, reproductive concerns, and psychosocial aspects. The correlations were classified as weak $(0-0.3)$, moderate $(0.3-0.7)$, and strong $(>0.7-1.0){ }^{22}$ To test the mediating effect of reproductive concerns, a path analysis was conducted using AMOS. The importance of parenthood was defined as an exogenous variable and depression and HRQoL as endogenous variables. Bootstrap procedures using 5000 samples were used to obtain the confidence intervals and significance of the indirect effects. ${ }^{23}$ The bias-corrected $95 \%$ bootstrap confidence intervals that do not include zero determined a significant mediation effect. To assess overall model fit, the chi-square statistics $\left(\chi^{2}\right)$, comparative fit index (CFI), standardized root mean residual (SRMR), and root mean square error of approximation (RMSEA) were used. Nonsignificant $\chi^{2}$ values and CFI equal to or greater than 0.95 , SRMR value below 0.08 and RMSEA below 0.06 were required for a goodfitting model. ${ }^{24}$

\section{Results}

\section{Descriptive statistics: sociodemographic, clinical,} and reproductive characteristics and concerns

Participants had an average age of 36.61 years (standard deviation $[\mathrm{SD}]=3.03$ ). Of the 104 respondents, $71.2 \%$ were married or lived in a cohabitation relationship and more than $50 \%$ had received college education. Most participants had received radiotherapy and $67.3 \%$ were undergoing endocrine therapy. The mean length of survival was 36.14 months $(\mathrm{SD}=24.51)$. Forty percent of the young women did not have a biological child and $43.3 \%$ reported they wanted a (another) child. At the time of enrolment in the study, 14 (13.5\%) participants had a history of miscarriage or abortion. Ninetytwo women $(88.5 \%)$ had received information about the implications of diagnosis/treatments for fertility, but only $19.2 \%$ reported having undergone fertility preservation before treatment. Women presented higher mean scores for concerns related to children's health risk and future life (mean $[\mathrm{M}]=3.90 ; \mathrm{SD}=1.12$ ) and to barriers to getting 
pregnant/having a child $(\mathrm{M}=2.76: \mathrm{SD}=0.95)$ compared with other concern of RCACS dimensions. Based on the cutoff criteria defined by Gorman et al., ${ }^{15} 35.6 \%$ of this sample presented moderate-to-high overall reproductive concerns. In addition, there is no relation between partnership status and reproduction-related concerns $(r=-1.78, p=0.071)$. Sociodemographic, clinical, and reproductive characteristics are presented in Table 1.

Table 1. Patient Sociodemographic, Clinical, AND ReProductive CharaCteristics $(N=104)$

\begin{tabular}{|c|c|c|c|c|}
\hline Characteristics & $\mathrm{n}$ & $\%$ & Mean & $S D$ \\
\hline Age at enrolment, in years & & & 36.61 & 3.03 \\
\hline Age at diagnosis, in years & & & 33.44 & 4.23 \\
\hline \multicolumn{5}{|l|}{ Relationship status } \\
\hline Married/cohabiting & 74 & 71.2 & & \\
\hline Single/other & 30 & 28.8 & & \\
\hline \multicolumn{5}{|l|}{ College educated } \\
\hline Yes & 54 & 51.9 & & \\
\hline No & 50 & 48.1 & & \\
\hline \multicolumn{5}{|l|}{ Employment status } \\
\hline Employed & 89 & 85.6 & & \\
\hline Unemployed & 13 & 12.5 & & \\
\hline Student & 1 & 1.0 & & \\
\hline Length of survival, in months & & & 36.14 & 24.51 \\
\hline \multicolumn{5}{|l|}{ Cancer treatment } \\
\hline Surgery & 89 & 85.6 & & \\
\hline Radiotherapy & 78 & 75.0 & & \\
\hline Endocrine therapy & 70 & 67.3 & & \\
\hline \multicolumn{5}{|l|}{ History of miscarriages/abortions } \\
\hline Yes & 14 & 13.5 & & \\
\hline No & 89 & 85.6 & & \\
\hline \multicolumn{5}{|l|}{ Number of biological children } \\
\hline No children & 42 & 40.4 & & \\
\hline One child or more & 62 & 59.6 & & \\
\hline \multicolumn{5}{|c|}{ Received information about fertility-related implications } \\
\hline Yes & 92 & 88.5 & & \\
\hline No & 12 & 11.5 & & \\
\hline \multicolumn{5}{|c|}{ Underwent fertility care before treatment } \\
\hline Yes & 20 & 19.2 & & \\
\hline No & 83 & 79.8 & & \\
\hline \multicolumn{5}{|l|}{ Wants a (or another) biological child } \\
\hline Yes & 45 & 43.3 & & \\
\hline No & 52 & 50.0 & & \\
\hline \multicolumn{5}{|l|}{ Overall reproductive concerns } \\
\hline Low $^{\mathrm{a}}$ & 67 & 64.4 & & \\
\hline Moderate to high $^{\mathrm{b}}$ & 37 & 35.6 & & \\
\hline \multicolumn{5}{|l|}{ Dimensions of concern } \\
\hline Fertility potential & & & 2.46 & 1.05 \\
\hline $\begin{array}{l}\text { Children's health risk and future } \\
\text { life }\end{array}$ & & & 3.90 & 1.12 \\
\hline Partner disclosure & & & 2.05 & 0.98 \\
\hline $\begin{array}{l}\text { Barriers to getting } \\
\text { pregnant/having children }\end{array}$ & & & 2.76 & 0.95 \\
\hline Acceptance & & & 2.31 & 0.90 \\
\hline
\end{tabular}

${ }^{\mathrm{a}}$ Mean scores in the RCACS $<3$.

${ }^{b}$ Mean scores in the RCACS $>3$ to 5 .

RCACS, Reproductive Concerns After Cancer Scale; SD, standard deviation.
Relationship between importance of parenthood, reproductive concerns, and psychosocial outcomes

There was a moderate positive correlation between importance of parenthood and self-reported reproductive concerns. Reproduction-related concerns were also positively associated with depression symptoms and negatively associated with physical, emotional, and social functioning. In turn, higher importance of parenthood was associated with higher levels of depression. Moderate negative correlations were found between depression and all functional scales of HRQoL (Table 2).

\section{Path analysis: the role of reproductive concerns}

A path model examining the indirect effect of the importance of parenthood on depression and HRQoL through reproductive concerns was conducted as shown in Figure 1. The importance of parenthood, overall reproductive concerns, and depression index were represented by single indicators. In turn, physical, emotional, and social functioning were specified as indicators of a single latent variable (unobserved) designated HRQoL. Based on correlations analysis, cognitive and role functioning were not included in the model since these variables were unrelated to reproductive concerns. Direct paths were controlled and dependent errors were correlated. The fit indexes indicated the good fit of the model $\left(\chi_{(6)}^{2}=5.83, p=0.443 ; \mathrm{CFI}=1.00, \mathrm{SRMR}=0.027\right.$, RMSEA $=0.000)$. As hypothesized, the effect of the importance of parenthood on HRQoL assessed through physical, emotional, and social functioning was fully mediated by reproductive concerns $(-0.133$ BC $95 \%$ CI: -0.274 to -0.034 , $p=0.013)$, that is, greater importance of parenthood in women's lives was associated with increased reproductive concerns and, consequently, they reported a poorer QoL. Additionally, there was a positive direct effect of the importance of parenthood on depression. However, no significant indirect effect was found in this relationship (0.032 BC $95 \%$ CI: -0.050 to $0.122, p=0.349$ ).

\section{Discussion}

To our knowledge, this is the first study providing preliminary evidence about the indirect effect of the meaning of parenthood on breast cancer patients' HRQoL, through reproductive concerns. These findings demonstrate the need to improve HRQoL, namely the physical, emotional, and social functioning of women who attach great importance to motherhood in their lives, by addressing reproductive concerns during health care provision.

In line with the results of Ljungman et al., ${ }^{9}$ the most common areas of concern among these young adult women were children's health risk and future life and the barriers to getting pregnant/having a child, involving issues related to their own health and future. Therefore, in addition to discussions about the effects of exposure to gonadotoxic therapy on the reproductive potential, concerns about perceptions of genetic risk and pregnancy as a trigger to recurrence should be addressed. As suggested in recent research, ${ }^{25}$ it is important to clarify misconceptions and to address preimplantation genetic diagnosis as an option for patients with a heritable syndrome. More specifically, the literature has shown that women with BRCA mutation have unique concerns related to 
Table 2. Pearson Correlations Between Importance of Parenthood, Reproductive Concerns, AND Psychosocial OUTCOMES

\begin{tabular}{|c|c|c|c|c|c|c|c|c|c|}
\hline Variables & $M e a n \pm S D$ & 1 & 2 & 3 & 4 & 5 & 6 & 7 & 8 \\
\hline 1. Reproductive concerns & $49.38 \pm 11.35$ & 1 & & & & & & & \\
\hline 2. Importance of parenthood & $58.36 \pm 15.30$ & $0.419 * * *$ & 1 & & & & & & \\
\hline 3. Depression & $3.42 \pm 3.42$ & $0.207 *$ & $0.343^{* * *} *$ & 1 & & & & & \\
\hline 4. Physical functioning & $82.58 \pm 14.54$ & $-0.228 *$ & -0.043 & $-0.546 * * *$ & 1 & & & & \\
\hline 5. Role functioning & $82.37 \pm 20.88$ & -0.110 & 0.013 & $-0.341 * * *$ & $0.523 * * *$ & 1 & & & \\
\hline 6. Emotional functioning & $71.37 \pm 26.34$ & $-0.193^{*}$ & -0.137 & $-0.486 * * *$ & $0.381 * * *$ & $0.267 * *$ & 1 & & \\
\hline 7. Cognitive functioning & $79.01 \pm 21.66$ & -0.179 & -0.034 & $-0.432 * * *$ & $0.508 * * *$ & $0.450 * * *$ & $0.547 * * *$ & 1 & \\
\hline 8. Social functioning & $78.37 \pm 26.57$ & $-0.255^{* *}$ & -0.176 & $-0.663 * * *$ & $0.429 * * *$ & $0.283^{* *}$ & $0.513 * * *$ & $0.370 * * *$ & $* 1$ \\
\hline
\end{tabular}

$* p<0.05$.

$* * p<0.01$

$* * * p<0.001$.

childbearing and feel pressure to make decisions about future motherhood. ${ }^{26}$ Special attention should be given to these issues, even when there is no personal history of cancer yet, but a germline mutation is present.

Our data suggested that a young adult with breast cancer who reported more need to start or complete their family and a more negative view of a childless life had a higher risk of experiencing depressive symptoms, regardless of their reproductive concerns. Although there is a gap in the literature regarding representations of the importance of parenthood and its impact on women with cancer, this direct effect is not completely surprising and similar results have been found in infertile women. ${ }^{27}$ These women have to deal with the uncertainty about their reproductive capacity and potential interruption or discontinuation of their family building projects. Particularly, when biological motherhood is a major goal in life, young adult women may find it more difficult to adjust their expectations, with acceptance and reorganization of a childless future resulting in more emotional difficulties. Interrupted desired childbearing is recognized as a distress factor, and even long-term survivors ${ }^{6}$ and some breast cancer patients, in the case of a hormone-sensitive disease, discontinue endocrine therapy due to their desire for future fertility. ${ }^{28}$ Based on this, it is imperative to fill the gaps in counseling about options to preserve fertility and alternative family-building options, ${ }^{5}$ decreasing psychological morbidity, and increasing the empowerment of these young adult women in their decision-making processes.

The major contribution of this study is to show the psychosocial impact of the importance of parenthood on the life of women faced with a reproduction-threatening disease. This study provided a better understanding of the psychological mechanisms involved in responding to the challenges of an oncological disease at a reproductive age, highlighting the mediating role of reproductive concerns. Future research should include larger samples and prospective longitudinal studies replacing cross-sectional designs, which do not reveal the trajectory of the meaning of parenthood and childlessness and its direct and indirect impact on psychosocial outcomes. A mixed-methods approach incorporating the voices of young adult women may also help explain the results of our path analysis. Additionally, the

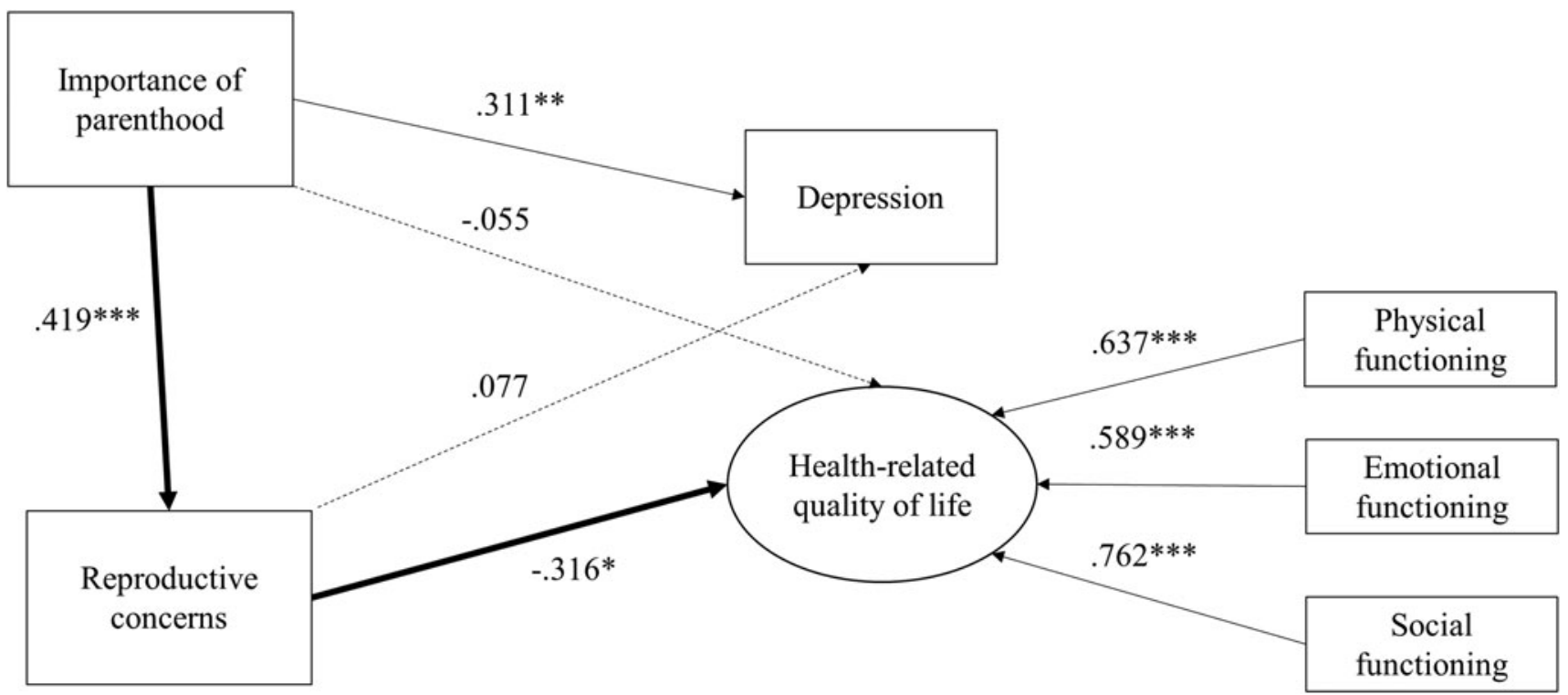

FIG. 1. Path model examining the mediating role of reproductive concerns. $*^{* *} p<0.001 ; * * p<0.01 ; * p<0.05$. Thick lines illustrate the mediation effect. 
length of survival and the use of fertility care should be considered in more complex pathway models.

\section{Author Disclosure Statement}

No competing financial interests exist.

\section{Funding Information}

This article was supported by National Funds through FCT-Fundação para a Ciência e a Tecnologia within CINTESIS, R\&D Unit (reference UID/IC/4255/2019); and a $\mathrm{PhD}$ fellowship for A.B. (SFRH/BD/115855/2016).

\section{References}

1. Ferlay J, Ervik M, Lam F, et al. Global cancer observatory: cancer today. Lyon, France: International Agency for Research on Cancer; 2018. Accessed July 17, 2019 from: https://gco.iarc.fr/today

2. Anchan RM, Ginsburg ES. Fertility concerns and preservation in younger women with breast cancer. Crit Rev Oncol Hematol. 2010;74(3):175-92.

3. Rosenberg SM, Partridge AH. Management of breast cancer in very young women. Breast. 2015;24(Suppl 2):S154-8.

4. Hubbeling HG, Rosenberg SM, González-Robledo MC, et al. Psychosocial needs of young breast cancer survivors in Mexico City, Mexico. PLoS One. 2018;13(5):e0197931.

5. Benedict C, Thom B, Friedman D, et al. Young adult female cancer survivors' unmet information needs and reproductive concerns contribute to decisional conflict regarding posttreatment fertility preservation. Cancer. 2016;122(13):2101-9.

6. van den Berg M, Baysal Ö, Nelen WLDM, et al. Professionals' barriers in female oncofertility care and strategies for improvement. Hum Reprod. 2019;34(6):1074-82.

7. Armuand GM, Wettergren L, Rodriguez-Wallberg KA, Lampic C. Desire for children, difficulties achieving a pregnancy, and infertility distress 3 to 7 years after cancer diagnosis. Support Care Cancer. 2014;22(10):2805-12.

8. Canada AL, Schover LR. The psychosocial impact of interrupted childbearing in long-term female cancer survivors. Psychooncology. 2012;21(2):134-43.

9. Ljungman L, Ahlgren J, Petersson LM, et al. Sexual dysfunction and reproductive concerns in young women with breast cancer: type, prevalence, and predictors of problems. Psychooncology. 2018;27(12):2770-7.

10. Villarreal-Garza C, Martinez-Cannon BA, Platas A, et al. Fertility concerns among breast cancer patients in Mexico. Breast. 2017;33:71-5.

11. Howard-anderson J, Ganz PA, Bower JE, Stanton AL. Quality of life, fertility concerns, and behavioral health outcomes in younger breast cancer survivors: a systematic review. J Natl Cancer Inst. 2012;104(5):386-405.

12. Peate M, Meiser B, Hickey M, Friedlander M. The fertilityrelated concerns, needs and preferences of younger women with breast cancer: a systematic review. Breast Cancer Res Treat. 2009;116(2):215-23.

13. Sobota A, Ozakinci, G. Fertility and parenthood issues in young female cancer patients-a systematic review. J Cancer Surviv. 2014;8(4):707-21.

14. Gorman JR, Malcarne VL, Roesch SC, et al. Depressive symptoms among young breast cancer survivors: the im- portance of reproductive concerns. Breast Cancer Res Treat. 2010;123(2):477-85.

15. Gorman JR, Su HI, Roberts SC, et al. Experiencing reproductive concerns as a female cancer survivor is associated with depression. Cancer. 2015;121(6):935-42.

16. Benedict C, Thom B, Friedman DN, et al. Fertility information needs and concerns post-treatment contribute to lowered quality of life among young adult female cancer survivors. Support Care Cancer. 2018;26(7):2209-15.

17. Gorman JR, Su HI, Pierce JP, et al. A multidimensional scale to measure the reproductive concerns of young adult female cancer survivors. J Cancer Surviv. 2014;8(2):218-28.

18. Bártolo A, Santos IM, Valério E, et al. Portuguese version of the reproductive concerns after cancer scale for young females. Psic Saúde Doenças. 2019;XX(Supplement):11.

19. Moura-Ramos M, Gameiro S, Canavarro MC, Soares I. Assessing infertility stress: re-examining the factor structure of the Fertility Problem Inventory. Hum Reprod. 2012; 27(2):496-505.

20. Pais-Ribeiro J, Silva I, Ferreira T, et al. Validation study of a Portuguese version of the Hospital Anxiety and Depression Scale. Psychol Health Med. 2007;12(2): 225-35.

21. Pais-Ribeiro J, Pinto C, Santos C. Estudo de validação da versão portuguesa do QLQ-C30-V.3. Psic Saúde Doenças. 2008;9(1):89-102.

22. Ratner B. The correlation coefficient: its values range between $+1 /-1$, or do they? J Target Meas Anal Market. 2009; 17(2):139-42.

23. Hayes AF. Beyond Baron and Kenny: statistical mediation analysis in the new millennium. Commun Monogr. 2009; 76(4):408-20.

24. $\mathrm{Hu} \mathrm{L}$, Bentler PM. Cutoff criteria for fit indexes in covariance structure analysis: conventional criteria versus new alternatives. Struct Equ Modeling. 1999;6(1):1-55.

25. Raghunathan NJ, Benedict C, Thom B, et al. Young adult female cancer survivors' concerns about future children's health and genetic risk. J Adolesc Young Adult Oncol. 2018;7(1):125-9.

26. Quinn GP, Vadaparampil ST, Tollin S, et al. BRCA carriers' thoughts on risk management in relation to preimplantation genetic diagnosis and childbearing: when too many choices are just as difficult as none. Fertil Steril. 2010;94(6):2473-5.

27. Moura-Ramos M, Gameiro S, Canavarro MC, et al. Does infertility history affect the emotional adjustment of couples undergoing assisted reproduction? the mediating role of the importance of parenthood. Br J Health Psychol. 2016;21(2):302-17.

28. Llarena NC, Estevez SL, Tucker SL, Jeruss JS. Impact of fertility concerns on tamoxifen initiation and persistence. J Natl Cancer Inst. 2015;107(10):djv202.

Address correspondence to: Ana Bártolo, MSc Department of Education and Psychology University of Aveiro Campus Universitário de Santiago Aveiro 3810-193 Portugal

E-mail: anabartolo@ua.pt 